\title{
GPPS-BJ-2019-0220
}

\section{Output-Feedback Model Predictive Control of Non-Ideal-Gas Heat Exchangers}

\author{
Viv Bone \\ University of Queensland \\ v.bone@uq.edu.au \\ Brisbane, QLD, Australia
}

\author{
Michael Kearney \\ University of Queensland \\ m.kearney@uq.edu.au \\ Brisbane, QLD, Australia
}

\author{
Thomas Reddell \\ University of Queensland \\ t.reddell@uq.edu.au \\ Brisbane, QLD, Australia
}

\begin{abstract}
Heat exchangers with non-ideal working fluids are core components of many industrial processes and power cycles, such as the supercritical CO2 Brayton cycle. In power applications, heat exchangers are involved in several control tasks. During steady operation, heat-exchanger outlet streams must be regulated to thermodynamic and mass flow setpoints in the presence of disturbances. During load-following operation, time-varying heat transfer profiles must be accurately tracked while respecting equipment and safety constraints. Model predictive control (MPC) can improve closed-loop performance in these scenarios since it performs well for transient multivariable control problems and can routinely deal with constraints.

Implementing MPC for non-ideal-gas heat exchangers is challenging due to 1) nonlinear fluid behaviours rendering simplified modelling techniques invalid, 2) inherent numerical limitations in simulating compressible-flow systems, and 3) the unavailability of fluid property measurements inside heat exchangers. We address these challenges by developing an MPC that is implemented via successive online linearisations of an analytical reduced-order 1D heat exchanger model. The controller's objective is to regulate the outlet state of the heat exchanger's process stream to a specified reference state. The setpoints of the compressors that drive mass flow through each stream are used as the control variables. A nonlinear observer based on this reduced-order model is used to estimate the internal state of the heat exchanger from inlet and outlet fluid property measurements. We perform closed-loop simulations for a CO2-CO2 heat exchanger and a molten salt-CO2 heat exchanger to analyse the performance of the controller. The controller provides good performance with prompt disturbance rejection and negligible steady-state offset.
\end{abstract}

\section{INTRODUCTION}

The supercritical CO2 (sCO2) Brayton cycle is a promising heat engine for next-generation thermal power plants due to its high thermal efficiency (Dostal et al., 2004), compatibility with several heat sources (Musgrove and Wright, 2017), compact and inexpensive turbomachinery (Turchi et al., 2013), and good scalability (Turchi et al., 2013). To achieve high efficiencies, sCO2 Brayton cycles need to be highly recuperated and thus require large heat exchangers. The heat exchangers in the sCO2 Brayton cycle are involved in several control tasks. During steady operation, the CO2streams at the outlets of the heater and gas cooler must be regulated to temperature, pressure, and mass flow rate setpoints in the presence of disturbances and equipment constraints. Breaching these constraints, for example, by overshooting the maximum turbine inlet temperature, risks damaging expensive equipment and causing plant downtime. Furthermore, sCO2 Brayton cycle plants may provide dispatchable power to metropolitan grids with high renewable penetration or to remote island-grid installations (Mehos et al., 2016). In these applications, sCO2 Brayton cycle plants will act in load-following roles, where control tasks are more complex. For example, to track a changing output power profile while maximising cycle efficiency, the CO2-stream outlet of the heater must be regulated to temperature and pressure setpoints while mass flow rate is modulated to meet output power requirements. In the aforementioned control scenarios, model predictive control (MPC) has the potential to improve plant performance since it performs well for transient multivariable control problems and it can routinely deal with equipment and safety constraints (Maciejowski, 2002).

To implement MPC for the heat exchangers in the sCO2 Brayton cycle, we require a control-oriented heat exchanger model that runs much faster than real time and is sufficiently 
accurate for good control performance (Lovera and Casella, 2015). Developing a model that meets these requirements is difficult for two reasons: 1) CO2 exhibits strong non-ideal gas effects over the operating conditions of interest (Dostal et al., 2004) and 2) compressible flow systems are inherently challenging to simulate due to numerical constraints on spatial and temporal discretisation. Computational fluid dynamics techniques can accurately simulate the dynamics of compressible flow systems like sCO2 heat exchangers, although they are too computationally expensive for control systems use. Data-driven approaches produce computationally efficient models, although they require large datasets to accurately capture the behaviour of complex nonlinear systems and have not been proven for transient simulations of compressible flow systems. Furthermore, datadriven approaches require specific training datasets for each component being modelled, limiting their practical application. Computationally-efficient dynamic models of sCO2 heat exchangers are best developed using 1D physicsbased approaches, where heat transfer behaviour and frictional pressure drop are captured using empirical correlations and fluid property variations are modelled using an appropriate equation of state (Moisseytsev and Sienicki, 2007; Carstens, 2007). The steady-state response of these models can be accurately calibrated to operational heat exchangers using only small experimental data sets (approximately 15 data points) (Bone et al., 2018).

$1 \mathrm{D}$ physics-based heat exchanger models solve the $1 \mathrm{D}$ Euler equations with source terms to account for heat transfer and frictional pressure drop, which are systems of nonlinear hyperbolic partial differential equations (PDEs) (LeVeque, 2002). For systems of hyperbolic PDEs, the different eigenmodes of the spatial differential operators contain approximately the same amount of energy, so the governing PDEs must be approximated by finite-dimensional ordinary differential equations (ODEs) to compute the dynamic behaviour of the system (Dubljevic et al., 2005). When explicitly solving these ODE approximations, as is required for MPC, the Courant-Friedrichs-Lewy (CFL) condition (Courant et al., 1967) must be satisfied. The CFL condition states that the maximum stable timestep is proportional to the speed of the fastest characteristic of the system of PDEs (Courant et al., 1967, which for the Euler equations is the downstream acoustic wave (Roe, 1986). For all flow regimes, this constraint on timestep renders real-time simulation of the 1D Euler equations for non-ideal gases computationally intractable on regular computers.

The computational efficiency of $1 \mathrm{D}$ heat exchanger models can be improved by using timescale separation to approximate the response of the fast subsystem, leaving only the dominant slow subsystem, where the characteristic speed is the bulk flow velocity. This approach significantly increases the allowable timestep, yielding a dynamic model that captures the phenomena releveant to control systems and that can run in real time. However, MPC requires a plant model that runs much faster than real time, so development of MPC based on this reduced-order nonlinear model is computational infeasible. Since the fastest dynamics of the reduced-order model are important for control, further timescale separation cannot be used to improve the model's speed. An alternative approach is to form a local approximation of the reduced-order nonlinear model that can be solved analytically, rather than numerically, thereby circumventing restrictions on timestep. Although the Euler equations and property variations of sCO2 are highly nonlinear, the reduced-order dynamic model is smooth, so there exists some neighbourhood around the current operating point where a linear model accurately approximates the true nonlinear model. Thus, for each MPC update, we compute a linear time invariant (LTI) model about the current operating point using a first-order Taylor series approximation and use this model as the basis for the controller. A discrete-time representation of this LTI model can be computed analytically, allowing the timestep to be selected solely on the desired update frequency, thereby making the solution of the MPC problem for sufficiently large prediction horizons computationally tractable. However, since the underlying system is highly nonlinear and it is difficult to analytically assess the accuracy of local LTI approximations, closed-loop simulations of test cases that are relevant to the sCO2 Brayton cycle are required to determine the validity and practical limitations of this control strategy.

\section{METHODOLOGY}

In this paper we develop a model predictive controller for the heat exchanger system shown in Fig. 1. The objective of the controller is to drive the outlet thermodynamic state and mass flow rate of one of the streams (the process stream) to specified reference values. The mass flow rate setpoints of the pumps/compressors are used as the control variables. We assume that fluid properties and mass flow rates are measured at the inlet and outlet of each stream.

We assess the controller's capabilities by performing closed-loop simulations where a high-fidelity simulation model is used to represent the 'true' behaviour of the plant. The controller is based on successive local linearisations of a simplified version of this underlying simulation model. We perform simulations for a CO2-CO2 heat exchanger and a solar salt (NaK)-CO2 heat exchanger that is representative of the heater in an sCO2 Brayton cycle.

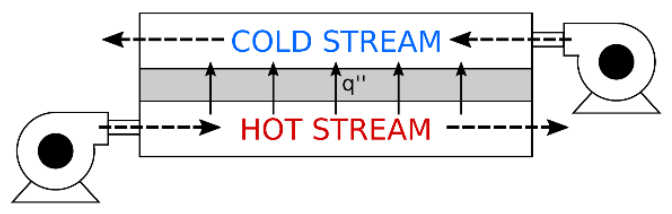




\section{Figure 1 - Schematic of the heat exchanger system}

\subsection{Simulation model}

Printed circuit heat exchangers (PCHEs) consists of several layers of plates into which zigzag-shaped microchannels have been chemically etched (Musgrove et al., 2017). Alternating hot-stream and cold-stream plates are arranged on top of one another in a counter-flow configuration and then diffusion bonded together to create a solid block. A plant-level simulation model for a PCHE can be developed by assuming that 1) mass flow is distributed evenly through all channels, 2) fluid properties in all channels are uniform at a given axial distance into the heat exchanger, and 3) all channels are geometrically identical. Under these assumptions, PCHEs may be modelled as two representative 1D fluid channels that are separated by a conductive wall. Each representative fluid stream is modelled using the quasi1D compressible flow equations, where fluid flow area, heat transfer area, and wall conduction area are scaled by the number of channels $N_{\text {channels }}$, and source terms are used to model frictional pressure drop and heat transfer to the channel wall. The source terms use Nusselt number and friction factor correlations to capture the effects of actual channel geometry and multidimensional fluid flow. Axial conduction within the fluid streams is neglected since it is typically negligible in PCHEs (Bone et al., 2018). The walls are also modelled as 1D elements since 1) axial conduction in PCHE walls is typically negligible and 2) the thermal resistance for cross-wall conduction is minimal compared to that for forced convection (Bone et al., 2018).

The governing equations for quasi-1D flow with frictional pressure drop and heat addition in a single representative fluid channel are

$$
\begin{gathered}
\text { Continuity } \frac{\partial \rho}{\partial t}=-\frac{\partial(\rho v)}{\partial x} \quad(2.1 \mathrm{a}) \\
\text { Momentum } \frac{\partial(\rho v)}{\partial t}=-\frac{\partial(\rho v|v|+p)}{\partial x}-f r \frac{\rho v|v|}{2 D_{H}} \\
\text { Energy } \frac{\partial(\rho E)}{\partial t}=-\frac{\partial(\rho H v)}{\partial x}-f r \frac{\rho v^{3}}{2 D_{H}}-\frac{q^{\prime \prime}}{A}
\end{gathered}
$$

where $E=e+0.5 v^{2}, H=h+0.5 v^{2}, D_{H}$ is the hydraulic diameter, $f r$ is Darcy friction factor, $A$ is the total flow area (i.e. for all channels), and $q^{\prime \prime}$ is the heat flux through the wall. Closure of the equations is achieved through an appropriate equation of state (see Sec. 2.7), which allows two known thermodynamic properties to be used to calculate all others. Wall temperature is governed by the ODE

$$
A_{w} \rho_{w} C_{p, w} \frac{d T_{w}}{d t}=q_{h}^{\prime \prime}+q_{c}^{\prime \prime},
$$

where $A_{w}, \rho_{w}, C_{p, w}$, and $T_{w}$ are the cross-sectional area, density, heat capacity, and temperature of the wall respectively, and $q_{h}^{\prime \prime}$ and $q_{c}^{\prime \prime}$ are the heat fluxes to the hot and cold stream. These heat fluxes are given by

$$
\begin{aligned}
& q_{h}^{\prime \prime}=N_{\text {channels }} U P_{h}\left(T_{h}-T_{w}\right) \\
& q_{c}^{\prime \prime}=N_{\text {channels }} U P_{c}\left(T_{c}-T_{w}\right),
\end{aligned}
$$

where $P_{h}$ and $P_{c}$ are the wetted perimeters of the hot and cold channels respectively and $U$ is the local heat transfer coefficient, which is calculated using the Nusselt number $\mathrm{Nu}$ and characteristic length $L_{C}$ as

$$
U=\mathrm{Nu} k / L_{C} \text {. }
$$

In the simulation model, we integrate Eqs. (2.1) over $N_{\text {cells,sim }}$ finite volumes spanning the computational domain, yielding a system of temporal ODEs for the evolution of density, velocity, and internal energy in each volume. For incompressible working fluids, density and pressure are by definition independent, and the discretised continuity equation becomes a constraint on the velocity field, rather than a transport equation for density (Versteeg and Malalasekera, 2007a). In this case, we use the unsteady PISO algorithm (Issa, 1986) on a staggered velocity grid with implicit Euler integration to compute the pressure and velocity fields. We then solve the unsteady energy equation with upwind differencing and implicit Euler integration to compute the temperature profiles at each timestep. We assume that fluid properties are fixed at the inflow boundary and assume fully developed flow at the outflow boundary.

For compressible working fluids, we directly solve the governing temporal ODEs using the AUSMDV flux splitting scheme (Wada, 1997) and explicit Euler time integration. The wall temperature ODEs are integrated over the same set of finite volumes and are solved using explicit Euler time integration. The inflow boundaries are modelled by assuming that the fluid isentropically accelerates from a reservoir at specified stagnation conditions into the computational domain, as done in the compressible flow CFD solver Eilmer4 (Jacobs and Gollan, 2017) for subsonic conditions. The reservoir stagnation conditions are modulated to achieve the desired mass flux at the inlet to the computational domain. The outflow boundary is modelled using the non-reflecting outflow scheme of the Navier-Stokes consistent boundary condition method (Poinsot and Lelef, 1992).

Numerical solution of Eqs. 2.1 using explicit methods is limited by the CFL condition (Courant et al., 1967),

$$
C=\frac{|u| \Delta t}{\Delta x} \leq C_{\max }
$$

where $\Delta t$ is timestep, $\Delta x$ is the spatial discretisation length, $u$ is the fastest characteristic, and $C_{\max }$ is a constant that takes the value 1 for explicit Euler time integration. Eqs. 2.1 share their homogenous part with the Euler equations, and therefore also share their characteristics, which are (Roe, 1986), 


$$
\begin{gathered}
u_{1}=v-a \\
u_{2}=v+a \\
u_{3}=v
\end{gathered}
$$

where $a$ is the speed of sound. The fastest characteristic, which governs the maximum stable timestep, is the downstream pressure wave (Eq. 2.6 b). When using explicit time integration methods, we set the timestep such that $C$ is approximately 0.9 .

The PISO algorithm is partially implicit and therefore not constrained by the CFL condition. Thus, for the incompressible flow model, we set the timestep such that $C$ is approximately 100 , primarily due to accuracy considerations.

\subsection{Control-oriented model}

The timestep restrictions imposed by the CFL condition on explicit time integration of Eqs. 2.1 render them unsuitable for control applications. However, since Mach number is typically less than 0.1 in PCHEs, the acoustic characteristics (Eqs. 2.6a-b) are generally much faster than the bulk-flow charactersitic (Eq. 2.6c). Thus, an appropriate control model can be developed by assuming that the acoustic dynamics stabilise infinitely quickly compared to the bulk-flow dynamics, thereby removing the fast characteristics and significantly relaxing the timestep restriction imposed by Eq. 2.5. This assumption also resolves the pressure-velocity coupling problem for incompressible fluids. Thus, the control model can be solved using the same numerical scheme for compressible and incompressible flow.

To develop a control-oriented model, we assume that the momentum dynamics are infinitely fast compared to the other dynamics (the quasi-steady momentum assumption), so that for the slow subsystem

$$
\frac{\partial(\rho v)}{\partial t}=0
$$

which implies that Eqn. 2.1b degenerates into the spatial ODE

$$
0=\frac{\partial(\rho v|v|+p)}{\partial x}+f r \frac{\rho v|v|}{2 D}
$$

In PCHEs, the pressure derivative usually dominates the momentum derivative, and thus Eq. 2.8can be approximated as

$$
\frac{\partial p}{\partial x}=-f r \frac{\rho v|v|}{\partial 2 D}
$$

Furthermore, since flow area is time invariant, under the quasi-steady momentum assumption, the continuity equation implies that mass flow rate through the heat exchanger is

$$
\dot{m}=\dot{m}_{\text {in }}
$$

where $\dot{m}_{i n}$ is the inlet mass flow rate. Using the chain rule, the time derivative in the energy equation (Eq. 2.1c) can be expressed as

$$
\frac{\partial(\rho E)}{\partial t}=\rho \frac{\partial E}{\partial t}+E \frac{\partial \rho}{\partial t} .
$$

Substituting the continuity equation (Eqn. 2.1a) into Eqn. 2.11 yields

$$
\frac{\partial(\rho E)}{\partial t}=\rho \frac{\partial E}{\partial t}-E \frac{\partial(\rho v)}{\partial x} .
$$

Since $\dot{m}$ is constant in space under the quasi-steady momentum assumption (Eq. 2.10) and since flow area does not vary along PCHEs

$$
\frac{\partial(\rho v)}{\partial x}=0,
$$

and thus the time derivative of total energy is given by

$$
\rho \frac{\partial E}{\partial t}=-\frac{\partial(\rho H v)}{\partial x}-f \frac{\rho v^{3}}{2 D}-\frac{q^{\prime \prime}}{A} .
$$

The frictional-loss term in Eq. 2.14 is typically negligible and since $v$ is typically small compared to $h$ and $e$, we assume that $H \approx h$ and $E \approx e$, so that

$$
\rho \frac{\partial e}{\partial t}=-\frac{\partial(\rho h v)}{\partial x}-\frac{q^{\prime \prime}}{A} \text {. }
$$

The governing equations for the control-oriented model are Eqs. 2.2, 2.9, and 2.15.

\subsection{Conversion to state-space form}

To implement MPC, we require that the control model consist of temporal ODEs in state-space form (Maciejowski, 2002). To obtain a model of this form, the governing equations for the control model are integrated over $N_{\text {cells }}$ finite volumes along the heat exchanger's length, yielding temporal ODEs for fluid energy and wall temperature and differential algebraic equations (DAEs) for pressure distribution.

Integrating the wall temperature ODE (Eq. 2.2) over a control volume yields

$$
\begin{aligned}
A_{w} \rho_{w} C_{p, w} \frac{d T_{w, i}}{d t}= & N_{\text {channels }}\left(U_{h} P_{C, h}\left(T_{h, i}-T_{w, i}\right)\right. \\
& \left.+U_{c} P_{C, c}\left(T_{c, i}-T_{w, i}\right)\right)
\end{aligned}
$$

where $T_{h, i}, T_{c, i}$, and $T_{w, i}$ are the temperatures in the $\mathrm{i}^{\text {th }}$ control volume for the hot stream, cold stream, and wall respectively. $U_{h}$ and $U_{c}$ are calculated according to Eq. 2.4, where all thermodynamic state variables have similarly been discretised into control volumes. $\Delta x_{i}$ has been cancelled from both sides of Eq. 2.16.

Integrating Eq. 2.15 over a control volume yields

$$
A \Delta x \rho_{i} \frac{d e_{i}}{t}=\left((\rho h v)_{i-\frac{1}{2}}-(\rho h v)_{i+\frac{1}{d 2}}\right)-\Delta x_{i} q^{\prime \prime}
$$


where the subscript $i-1 / 2$ indicates the flux value at the interface between cells $i-1$ and $i$ and similar for $i+1 / 2$. For systems where convection dominates diffusion, such as PCHEs, using central differencing to compute fluxes results in numerical instability (Versteeg and Malalasekera, 2007b). Thus, for simplicity, we use upwind differencing to compute flux values,

$$
\begin{array}{llll}
\phi_{i+\frac{1}{2}} & =\phi_{i} & \phi_{i-\frac{1}{2}}=\phi_{i-1} & \text { for } v \geq 0 \\
\phi_{i+\frac{1}{2}}=\phi_{i+1} & \phi_{i-\frac{1}{2}}=\phi_{i} & \text { for } v<0,
\end{array}
$$

where $\phi$ is an arbitrary quantity. For positive $v$ this gives

$$
\frac{d e_{i}}{d t}=\frac{m_{i n}\left(h_{i-1}-h_{i}\right)}{d A \Delta x \rho_{i}}-\frac{\mathrm{Nu} k_{i}}{d_{\text {channel }}^{2} \rho_{i}}\left(T_{i}-T_{w, i}\right)
$$

where $d_{\text {channel }}$ is the diameter of single PCHE channel. Any appropriate explicit Nusselt number correlation may be substituted into Eq. 2.19.

The spatial ODE arising directly from the quasi-steady momentum assumption (Eq. 2.9) is converted to an explicit DAE as follows. Firstly, for rough channels, $f r$ is given by the implicit Colebrook-White equation (White, 2011). Explicit approximations for $f r$ are available, however all are functions of Reynolds number, which must be computed implicitly using an equation of state for non-ideal fluids. Thus, to obtain an explicit control model, we assume that the axial distribution of $f r$ persists over the prediction horizon and compute future estimates of $\rho$ and $v$ using a Taylor series expansion around the current time $t_{0}$ and current fluid state $\left(e_{0}, p_{0}\right)$ as Thus, to obtain an explicit control model, we assume that the axial distribution of $\mathrm{fr}$ persists over the prediction horizon and estimate $\rho$ at $k$ timesteps into the future using a Taylor series expansion around the current time $t_{0}$ and current fluid state $\left(e_{0}, p_{0}\right)$ as

$\rho_{k}=\rho_{0}+\left.\frac{\partial \rho}{\partial e}\right|_{p_{0}, t_{0}}\left(e_{k}-e_{0}\right)+\left.\frac{\partial \rho}{\partial p}\right|_{e_{0}, t_{0}}\left(p_{k}-p_{0}\right)$.

Future velocity estimates are obtained from these density estimates and future mass flow rate estimates (see Sec. 2.4) as

$$
v_{k}=\frac{\dot{m}_{k}}{\rho_{k}} \frac{1}{A}
$$

Under the quasi-steady momentum assumption, future estimates pressure estimates are obtained from $v_{k}, \rho_{k}$, and future inlet pressure estimates $p_{0, k}$ by integrating Eq. 2.9 over $N_{\text {cells }}$ finite volumes, yielding

$$
p_{i+1, k}=p_{i, k}-f_{i, k} \frac{\Delta x_{i, k} \rho_{i, k} v_{i, k}^{2}}{2 D_{H}},
$$

where $i$ indexes the cells spatially.
To obtain a state-space model, we choose thermodynamic state variables of $e$ and $\rho$ for the control-oriented model and compute all other thermodynamic properties in Eqs. 2.16, 2.19, and 2.22 using an appropriate equation of state (see Sec. 2.7).

\subsection{Pump and compressor modelling}

The inlet mass flow rate of each heat exchanger stream is set by a pump for incompressible fluids and a compressor for compressible fluids. For incompressible fluids, the thermodynamic state at the pump outlet is constant and the mass flow rate is linearly proportional to pump speed. For compressible fluids, the efficiency and pressure ratio of the compressor are functions of the speed, guide vane angle, and inlet thermodynamic state, so the compressor outlet thermodynamic state varies with mass flow rate in a complex nonlinear manner. Here, however, we take a simplified approach by assuming that compressor efficiency and pressure ratio are constant for all speeds and thus all mass flow rates that we model. Under these assumptions, the outlet thermodynamic conditions of the compressor are constant and the mass flow rate varies linearly with compressor speed. This simplified approach introduces some error in the compressor model but is satisfactory for investigating the feasibility of controlling real-gas heat exchangers with MPC.

We consider the case where a PID controller modulates pump or compressor speed such that mass flow rate is driven to the commanded setpoint $\dot{m}_{r e f}$. We do not explicitly model the separate pump and PID controller subsystems; instead, we assume that the closed-loop dynamics of the complete system can be modelled by a linear second-order ODE,

$$
I \frac{d^{2} \dot{m}}{d t^{2}}=k\left(\dot{m}_{r e f}-\dot{m}\right)-c \frac{d \dot{m}}{d t}
$$

and that some PID gains exist that give the desired closed-loop performance ( $I, c$, and $k$ are the effective inertia, damping coefficient, and spring constant of the system). We set the natural frequency to a value of $2 \mathrm{~Hz}$ in accordance with typical operating data and assume that the PID is tuned to give a slightly overdamped response with a damping ratio of 1.3. Additionally, we consider that the mass flow rate has an upper limit $\dot{m}_{\text {max }}$ and a maximum rate of change in both direction $d \dot{m} / d t_{\max }$ that are dictated by maximum speed and rotordynamics respectively.

\subsection{Linearisation}

MPC requires that the plant model take the explicit statespace form,

$$
\begin{aligned}
\frac{d x}{d t} & =f(x, u) \\
y & =g(x, u)
\end{aligned}
$$


where $x, u$, and $y$ are the state, control and output vectors of lengths $n, l$, and $m$ respectively, $f($.$) is the nonlinear control-$ oriented model, and $g($.$) is a function that computes the$ measured outputs from the state and control vectors. For realgas heat exchangers, $f$ is comprised of Eqs. 2.16, 2.19, and 2.22 for the fluid stream dynamics, Eq. 2.16 for the wall dynamics, and Eq. 2.23 for the

pump/compressor dynamics. $\mathrm{x}$ is comprised of the hot-stream and cold-stream thermodynamic states, wall temperatures in each axial discretisation, and mass flow rates and their derivatives at the outlet of each pump/compressor, so

$=\left[e_{\text {hot }}, p_{\text {hot }}, e_{\text {cold }}, p_{\text {cold }}, T_{\text {wall }}, \dot{m}_{\text {hot }}, \frac{d \dot{\mathrm{m}}_{\text {hot }}}{d t}, \dot{\mathrm{m}}_{\text {cold }}, \frac{d \dot{\mathrm{m}}_{\text {cold }}}{d t}\right]^{\top}$,

where

$$
e_{\text {hot }}=\left[e_{\text {hot }, 1}, \ldots, e_{\text {hot }, N_{\text {cells }}}\right]^{\top}
$$

and similar for the other thermodynamic properties. For the case that we study, the mass flow rate setpoints for each stream are used as the control inputs, so

$$
u=\left[m_{\text {ref }, \text { hot }}, m_{\text {ref,cold }}\right] \text {. }
$$

$\mathrm{y}$ is specific to the particular operating case being analysed and is discussed in Sec. 3.2. A nonlinear explicit state-space model may be obtained from Eqs. 2.16, 2.19, and 2.22 by developing explicit approximations of the equation of state, however, even under the quasi-steady momentum approximation (Eq. 2.7), this model would be severely limited in allowable timestep due to the CFL condition (Eq. 2.5). Additionally, solving predictive control problems for nonlinear systems with high state dimensionality, such as real-gas heat exchangers, is computationally prohibitive. Thus, at each timestep, we compute a local linear approximation of the nonlinear model about the current operating point $\left(x_{0}, u_{0}\right)$ and use this linear model to implement the controller.

A linear approximation to the nonlinear model is developed by considering the effects of small perturbations in the state and control variables,

$$
\begin{aligned}
& \bar{x}=x-x_{0} \\
& \bar{u}=u-u_{0} .
\end{aligned}
$$

Using these perturbed variables, Eqn. 2.24 may be approximated using a Taylor series as

$$
\begin{aligned}
\frac{d x}{d t} & =f\left(x_{0}+\bar{x}, u_{0}+\bar{u}\right) \\
& \approx f\left(x_{0}, u_{0}\right)+\left.\frac{\partial f}{\partial \bar{x}}\right|_{x_{0}, u_{0}} \bar{x}+\left.\frac{\partial f}{\partial \bar{u}}\right|_{x_{0}, u_{0}} \bar{u} \\
y & \approx g\left(x_{0}, u_{0}\right)+\left.\frac{\partial g}{\partial \bar{x}}\right|_{x_{0}, u_{0}} \bar{x}+\left.\frac{\partial g}{\partial \bar{u}}\right|_{x_{0}, u_{0}} \bar{u}
\end{aligned}
$$

where quadratic and higher order terms have been neglected. By noting that

$$
\frac{d x}{d t}=\frac{d \bar{x}}{d t}
$$

we obtain an approximate linear time-invariant (LTI) statespace model about the current operating point $\left(x_{0}, u_{0}\right)$ in the new variables $\bar{x}$ and $\bar{u}$

$$
\begin{aligned}
\frac{d \bar{x}}{d t} & =A \bar{x}+B \bar{u}+f_{0} \\
y & =y_{0}+C \bar{x}+D \bar{u}
\end{aligned}
$$

where

$$
\begin{gathered}
A=\left.\frac{\partial f}{\partial \bar{x}}\right|_{x_{0}, u_{0}}, B=\left.\frac{\partial f}{\partial \bar{u}}\right|_{x_{0}, u_{0}}, f_{0}=f\left(x_{0}, u_{0}\right) \\
C=\left.\frac{\partial g}{\partial \bar{x}}\right|_{x_{0}, u_{0}}, D=\left.\frac{\partial g}{\partial \bar{u}}\right|_{x_{0}, u_{0}}, y_{0}=g\left(x_{0}, u_{0}\right) .
\end{gathered}
$$

The matrices $A, B, C$, and $D$ are determined using a computer algebra system and their terms are excluded here for brevity. Terms in these matrices contain fluid property partial derivatives which can be obtained directly from fluid property databases such as CoolProp (Bell et al., 2014). Partial derivatives of Nusselt number correlations may be determined analytically or numerically. Since Nusselt number correlations for turbulent flow are always functions of Reynolds number, partial derivatives with respect to mass flow rate and the two thermodynamic state variables must be included. Commonly, the nonlinear model is linearised about an equilibrium point, making $f_{0}$ zero (Maciejowski, 2002). However, due to the highly nonlinear nature of real-gas heat exchangers, the linearisation must be frequently recomputed, often about nonequilibrium points.

\subsection{Conversion to discrete time}

To implement MPC, the linearised plant model must be converted to discrete-time form,

$$
\begin{gathered}
\bar{x}_{k+1}=A_{d} \bar{x}_{k}+B_{d} \bar{u}_{k}+f_{0 d} \\
y_{k}=y_{k}+C \bar{x}_{k}+D \bar{u}_{k},
\end{gathered}
$$

where $A_{d}, B_{d}$, and $f_{0, d}$ are the discrete-time versions of the $A$ and $B$ matrices and $f_{0}$ vector. Discrete time conversion is performed by noting that $f_{0}$ is time-invariant until the model is re-linearised and by using a zero-order hold assumption for $\bar{u}$. This assumption on $\bar{u}$ is valid since the control inputs are the mass flow rate setpoints rather than the mass flow rates themselves. Under these conditions, analytic solutions for the discrete-time LTI matrices are

$$
\begin{gathered}
A_{d}=\exp (A \Delta t) \\
B_{d}=\int_{0}^{\Delta t} \exp (A(\Delta t-\tau)) d \tau B
\end{gathered}
$$




$$
\Gamma_{d}=\int_{0}^{\Delta t} \exp (A(\Delta t-\tau)) d \tau I
$$

where $\Delta t$ is the sampling interval (Franklin et al., 1990) and $\Gamma_{d}$ is the discrete-time coefficient matrix of $f_{0}$ such that

$$
f_{0 d}=\Gamma_{d} f_{0}
$$

$A_{d}, B_{d}$, and $f_{0, d}$ are evaluated using the properties (DeCarlo, 1989)

$$
\begin{aligned}
& \exp \left(\left[\begin{array}{cc}
A & B \\
\overline{0} & \overline{0}
\end{array}\right] \Delta t\right)=\left[\begin{array}{cc}
A_{d} & B_{d} \\
\overline{0} & I
\end{array}\right] \\
& \exp \left(\left[\begin{array}{cc}
A & \frac{I}{0}
\end{array}\right] \Delta t\right)=\left[\begin{array}{cc}
A_{d} & \Gamma_{d} \\
\overline{0} & I
\end{array}\right],
\end{aligned}
$$

where $\overline{0}$ denotes zero matrices sized such that the arguments of the matrix exponentials in Eq. 2.35 are square. $A$ is typically poorly conditioned, so the matrix exponentials in Eq. 2.35 are computed using the scaling and squaring algorithm (Moler and Van Loan, 2003) with a $13^{\text {th }}$ order Padé approximant, which is evaluated using Horner's method (Higham, 2005). Since the expressions for the discrete-time matrices given in Eq. 2.33 are exact, the resulting model is a highly accurate representation of the continuous-time LTI at the sampling instants $k \Delta t$. Additionally, since the discrete-time matrices are not computed using a temporal discretisation method, the CFL condition need not be obeyed and an arbitrarily large $\Delta t$ may be chosen, thereby allowing the use of a suitably large prediction horizon.

\subsection{Fluid property calculations}

We calculate all fluid properties using lookup tables generated using the open-source fluid property database CoolProp (Bell et al., 2014). CoolProp computes CO2's thermodynamic state by iteratively solving the Span and Wagner equation of state (Span and Wagner, 1996), and computes its thermal conductivity and viscosity using the Scalabrin et. al. (Scalabrin et al., 2006) and Fenghour et. al. (Fenghour et al.,1998) correlations respectively. CoolProp models sodium nitrate (known as 'solar salt') using an incompressible equation of state that is based on experimental data from Zavoico (Zavoico, 2001).

\section{CONTROL STRATEGY}

\subsection{State estimation}

To implement MPC, the full state vector $x$ must be known at the current timestep $k$ (Maciejowski, 2002). We assume that the outlet mass flow rate from each pump/compressor and its derivative (arising in Eq. 2.23) are measured exactly using appropriate flow meters. For heat exchangers, however, the internal thermodynamic state profiles are not directly measurable and must be estimated using an observer. We consider the case where the inlet and outlet thermodynamic states are measured exactly using ideal temperature and pressure sensors. For this case, the vector of measured outputs is

$y \quad$ (3.1)

$=\left[e_{\text {out }, \text { hot }}, p_{\text {out }, \text { hot }}, e_{\text {out }, \text { cold }}, p_{\text {out }, \text { cold }}, \dot{m}_{\text {hot }}, \frac{d \dot{m}_{\text {hot }}}{d t}, \dot{m}_{\text {cold }}, \frac{d \dot{m}_{\text {cold }}}{d t}\right]^{\top}$.

The outlet properties in $y$ may be calculated by extrapolating over half a cell from the first or last cell values in $x$ using onesided finite differences. Using this approach with secondorder differencing, the cold-stream outlet internal energy is computed as

$$
\begin{gathered}
e_{\text {out }, \text { cold }}=e_{\text {cold }, 1}-0.5 \Delta x \frac{d e_{\text {cold }}}{d x} \\
e_{\text {out }, \text { cold }}=1.75 e_{\text {cold }, 1}-1.0 e_{\text {cold }, 2}+0.25 e_{\text {cold }, 2},
\end{gathered}
$$

and similar for pressure, and for the hot-stream outlet conditions. This extrapolation is a linear function of $x$, so

$$
g(\hat{x}, u)=C x
$$

where to record the outlet conditions of each stream, C includes the nonzero entries

$$
\begin{array}{ccc}
C_{1, N_{\text {cells }}}, C_{2,2 N_{\text {cells }}}=1.75, & C_{1, N_{\text {cells }}-1}, C_{2,2 N_{\text {cells }}-1}=-1.0, & C_{1} \\
C_{3,2 N_{\text {cells }}+1}, C_{4,3 N_{\text {cells }}+1}=1.75 & C_{3,2 N_{\text {cells }}+2}, C_{4,3 N_{\text {cells }}+2}=-1.0, & C_{3,}
\end{array}
$$

and to record the mass flow rate terms,

$C_{5,4 N_{\text {cells }}+1}, C_{5,4 N_{\text {cells }}+2}, C_{5,4 N_{\text {cells }}+3}, C_{5,4 N_{\text {cells }}+4}=1$.

Additionally, the observer is augmented with an integrating disturbance model to account for mismatch between the simulation and control models. The disturbance model drives the output estimates to zero as $t \rightarrow \infty$, thereby ensuring that state estimate converges. Since the state estimate converges, the controller can track a constant reference without steady-state offset (Borrelli et al, 2017).

A nonlinear observer that estimates $\hat{x}$ from $y$ takes the form

$$
\begin{array}{cc}
\frac{d \hat{x}}{d t} & =f(\hat{x}, u)+L(y-\hat{y}) \\
\hat{y} & =g(\hat{x}, u)+\hat{d} \\
& \hat{d}_{k+1}=\hat{d}_{k}+L_{d}\left(y_{k}-\hat{y}_{k}\right)
\end{array}
$$

where $f($.$) is the reduced-order nonlinear model (Eqs. 2.16,$ 2.19, 2.22, 2.16, and 2.23, see Secs. 2.2-2.4), 'hats' indicate estimated quantities, and $L$ is the $n \times m$ observer gain matrix. The reduced-order model, rather than the full simulation model, is used since the observer must run in real time. To compute updated state estimates, Eq. 3.7 is solved using explicit Euler integration with the Courant number equal to 0.9 , with the speed of the fastest characteristic being given by Eq. 2.6c. All elements in the matrix $L_{d}$ are set to 0.1. 
The observer gain matrix $L$ is set using the following logic. We assume that the sensors have no error, so the inlet thermodynamic state for each stream is known exactly. Since heat transfer is a cumulative process, we assume that the errors in the estimates for $e$ and $p$ for each stream gradually increase from zero to their maximum value as fluid travels from the inlet to the outlet. We use only the error in the outlet value of a given fluid property to correct internal estimates for that property (for example, we use only $e_{\text {cold,out }}-\hat{e}_{\text {cold,out }}$ to correct the estimates of $e$ along the cold stream). Since the error in the state estimate gradually increases from inlet to outlet, we scale the observer gains using a linear function from zero at the inlet to unity at the outlet. Thus, the observer gains corresponding to the state estimate at cell $i$ for the cold stream are

$$
L_{i, 1}, L_{i+N_{\text {cells }, 2}}=\frac{i-0.5}{N_{\text {cells }}} \times k_{o b s},
$$

and the gains corresponding to the hot stream state estimates are

$$
L_{i+2 N_{\text {cells }}, 3}, L_{i+3 N_{\text {cells }}, 4}=\frac{N_{\text {cells }}-i+0.5}{N_{\text {cells }}} \times k_{\text {obs }},
$$

where $k_{\text {obs }}$ is a tuning parameter for the observer dynamics. A value of $5 \times 10^{-4}$ was found to be appropriate for $k_{\text {obs }}$. All other values in $L$ are zero. The observer does not directly correct wall temperature estimates since the wall temperatures are not measured at any location. However, since the wall temperatures are governed by adjacent fluid temperatures, they are indirectly corrected by the observer through the reduced-order heat exchanger model.

\subsection{MPC formulation}

We develop a model predictive controller that drives the process-stream outlet of a heat exchanger to a specified temperature and mass flow rate using the mass flow rate setpoints of both streams as the control inputs. We consider the case where the process stream is the cold stream and where the heat exchanger is arranged in counter-flow configuration with the cold-stream outlet at a nondimensional distance of $d=0$ and the hot-stream outlet at $d=1$. Here, the vector of controlled outputs is

$$
z=\left[e_{\text {out }, \text { cold }}, \dot{m}_{\text {cold }}\right] .
$$

Additionally, we assume that the component connected to the cold-stream outlet has a maximum temperature limit Tmax that must be respected by the controller.
Tracking MPC with move suppression (Maciejowski, 2002) is used to drive the controlled outputs to their specified target values $r$. For tracking MPC, the cost function takes the form

$$
\begin{array}{r}
J(k, x, \mathbf{u})=\sum_{i=1}^{H_{p}}\left(\hat{z}_{k+i}-r\right)^{\top} Q\left(\hat{z}_{k+i}-r\right) \\
+\sum_{i=0}^{H_{u}}\left(\Delta u_{k+i}\right)^{\top} R\left(\Delta u_{k+i}\right),
\end{array}
$$

where $\boldsymbol{u}$ is the vector of control inputs over the control horizon

$$
\mathbf{u}=\left[u_{k}, u_{k+1}, \ldots, u_{k+H_{u}}\right]
$$

$\Delta u_{k+1}=u_{k+i}-u_{k+i-1}, Q$ is the weighting matrix for the outputs, and $R$ is the move suppression matrix ( $Q$ and $R$ are both diagonal). So that tracking errors for each controlled output are weighted approximately equally, we set the entries of $Q$ using the nominal values of each of the controlled outputs (denoted using overbars) as

$$
Q=\operatorname{diag}\left(\bar{e}_{\text {out }, \text { cold }}^{-1}, \overline{\dot{m}}_{\text {cold }}^{-1}\right)
$$

For moderate move suppression, we set

$$
R=\operatorname{diag}(0.5,0.5) \text {. }
$$

The maximum cold stream outlet temperature limit $T_{\text {max }}$ is incorporated into the MPC by computing $e_{\text {cold,max }}-$ the internal energy equivalent to $T_{\max }$ at the current coldstream outlet pressure - then imposing the output constraint

$$
e_{\text {out,cold }} \leq e_{\text {cold,max }}
$$

at all timesteps. Additionally, assuming that reverse flow is not permissible, the constraints on mass flow rate imposed by the turbomachinery (see Sec. 2.4) are incorporated into the MPC formulation by imposing the input constraints

$$
\begin{aligned}
& 0 \leq \dot{\mathrm{m}}_{\text {hot }} \leq \dot{\mathrm{m}}_{\text {hot,max }}, \\
& -{\frac{d \dot{\mathrm{m}}_{\text {hot }}}{d t}}_{\text {max }} \leq \frac{\dot{\mathrm{m}}_{\text {hot }}}{d t} \leq{\frac{d \dot{\mathrm{m}}_{\text {hot }}}{d t}}_{\text {max }},
\end{aligned}
$$

and analogous for the cold stream.

At each sampling instant $k$, to compute the next control update, the constrained quadratic programming problem

$$
\begin{array}{cc}
\min _{\mathbf{u}} & J(k, \hat{x}, \mathbf{u}) \\
\text { subject to } & \bar{x}_{k+1}=A_{d} \bar{x}_{k}+B_{d} \bar{u}_{k}+f_{0 d} \\
\hat{y} \in\left[y_{\min }, y_{\max }\right] \\
u \in\left[u_{\min }, u_{\max }\right] \\
\Delta u \in\left[\delta u_{\text {min }}, \delta u_{\max }\right],
\end{array}
$$

is solved for the current state estimate $\hat{x}$ using the constrained optimisation software Gurobi (Gurobi Optimization, 2018). Only $u_{k}$ from $\boldsymbol{u}$ is used as the control signal, and at the next 
sampling instant, the process is repeated to compute the subsequent control update. Due to its high state dimensionality, we formulate the optimisation problem using dense matrices, so its time complexity depends only its input and output dimensionalities (Wang and Boyd, 2010).

\section{RESULTS}

We test the performance of the MPC developed in Sec. 3 by performing closed-loop simulations of a CO2-CO2 heat exchanger and a NaK-CO2 heat exchanger. Both heat exchangers have a length of $1 \mathrm{~m}, 1600$ channels per stream, a channel diameter of $1.06 \mathrm{~mm}$, and a wall thickness of $0.6 \mathrm{~mm}$. We use the Ngo correlation, $\mathrm{Nu}=0.1696 \mathrm{Re}^{0.629} \mathrm{Pr}^{0.317}$ (Ngo et al., 2007), to model heat transfer in CO2 streams and the correlation for fully-developed laminar flow in a straight channel with constant heat flux, $N u=4.089$ (Faghri et al., 2010), to model heat transfer in the salt streams.

We simulate the closed-loop behaviour of the $\mathrm{CO} 2-\mathrm{CO} 2$ heat exchanger in response to two load changes, one occurring close to design-point conditions and one occurring at highly off-design conditions in the presence of inlet temperature disturbances. We perform a single simulation around designpoint conditions for the salt-CO2 heat exchanger. We simulate the behaviour of the plant using the simulation model described in Sec. 2.1 with $N_{\text {cells,sim }}=100$. We compute state estimates at $1 \mathrm{kHz}$ using the nonlinear observer developed in Sec. 3.1 with $N_{\text {cells }}=12$. We use these state estimates to compute control updates at $4 \mathrm{~Hz}$ using the MPC developed in Sec. 3.2, also with $N_{\text {cells }}=12$ and with prediction and control horizons of $H_{p}, H_{u}=40$ (10 sec).

For the design-point $\mathrm{CO} 2-\mathrm{CO} 2$ case, we simulate the behaviour of the controlled heat exchanger system in response to setpoint changes in cold-stream outlet temperature from $635 \mathrm{~K}$ to $700 \mathrm{~K}$ and cold-stream mass flow rate from $5.55 \mathrm{~kg} / \mathrm{s}$ to $3.2 \mathrm{~kg} / \mathrm{s}$ at $\mathrm{t}=1 \mathrm{sec}$ over $15 \mathrm{sec}$. The initial and final temperature distributions are shown in Fig. 2 and the temporal profiles of inlet and outlet temperature and mass flow rate for both streams are shown in Fig. 3. For the off-design CO2-CO2 case, we simulate the system's response to setpoint changes in cold-stream outlet temperature from $435 \mathrm{~K}$ to $550 \mathrm{~K}$ and coldstream mass flow rate from $5.55 \mathrm{~kg} / \mathrm{s}$ to $3.2 \mathrm{~kg} / \mathrm{s}$ at $\mathrm{t}=0 \mathrm{sec}$ over 15 sec. In this test case, the inlet temperatures of both streams are subject to disturbances beginning at $t=6 \mathrm{sec}$. The initial and final temperature distributions are shown in Fig 4. and the temporal profiles of inlet and outlet temperature and mass flow rate are shown in Fig. 5.
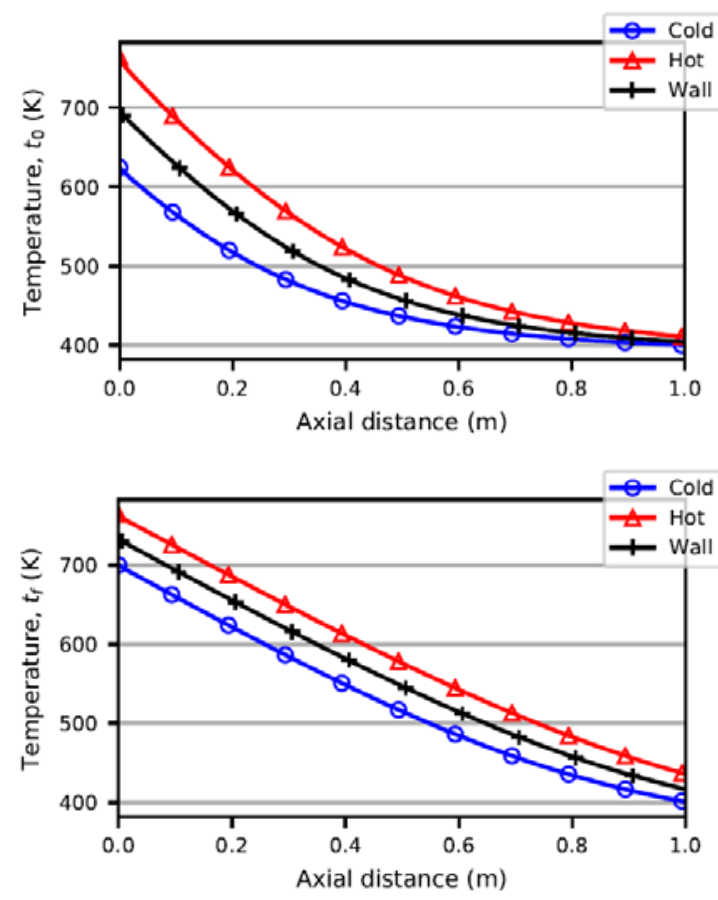

Figure 2 - Initial $\left(t_{0}\right)$ and final $\left(t_{f}\right)$ temperature profiles for the design-point $\mathrm{CO}$ - $\mathrm{CO} 2$ case
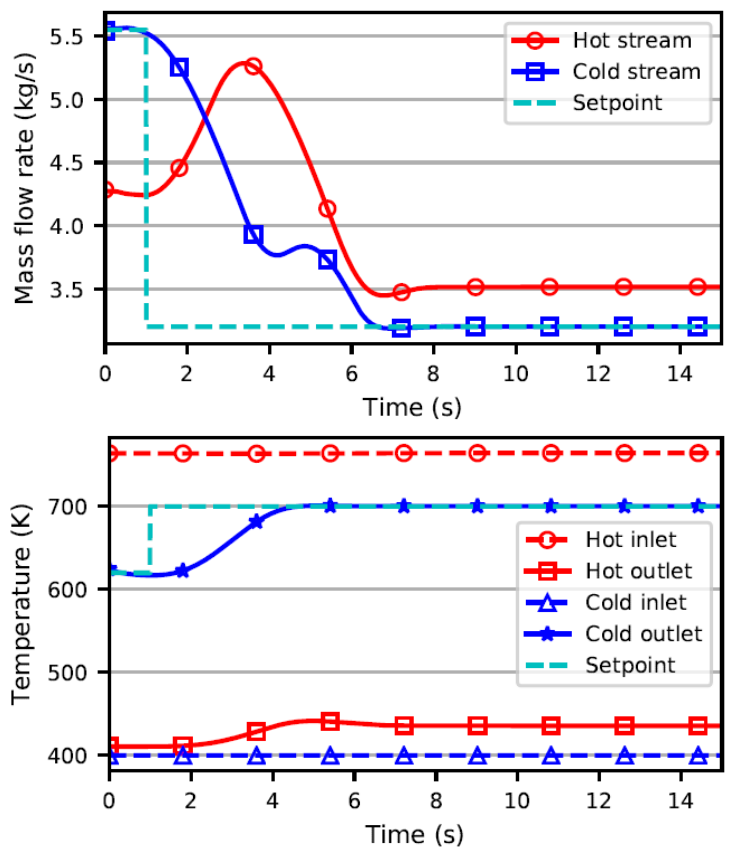

Figure 3 - Closed-loop response of inlet and outlet temperatures and mass flow rates for the designpoint CO2-CO2 case 

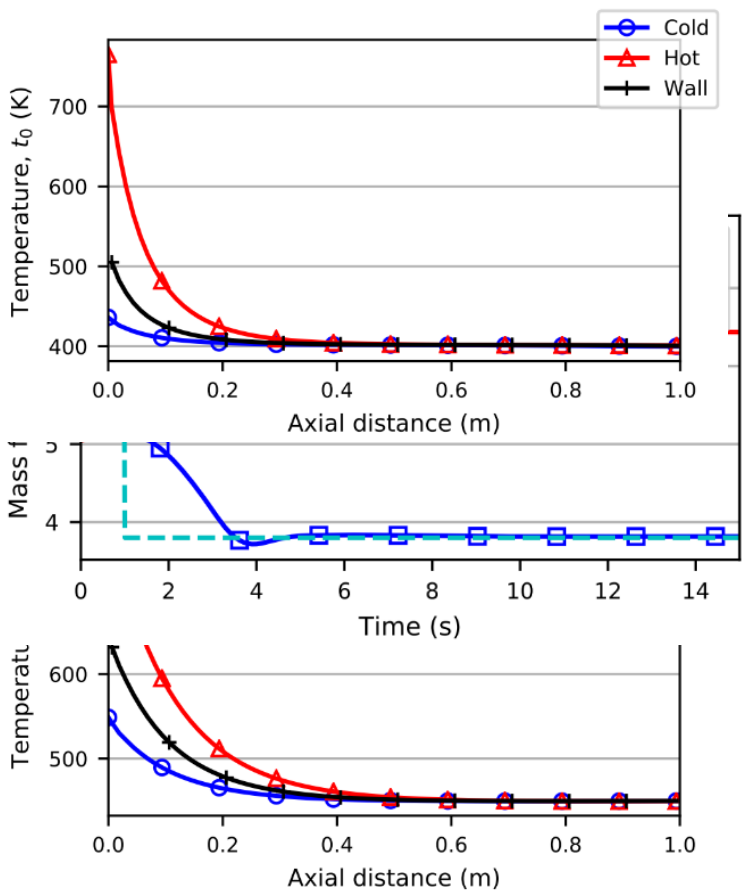

Figure 4 - Initial $\left(t_{0}\right)$ and final $\left(t_{f}\right)$ temperature profiles for the off-design case

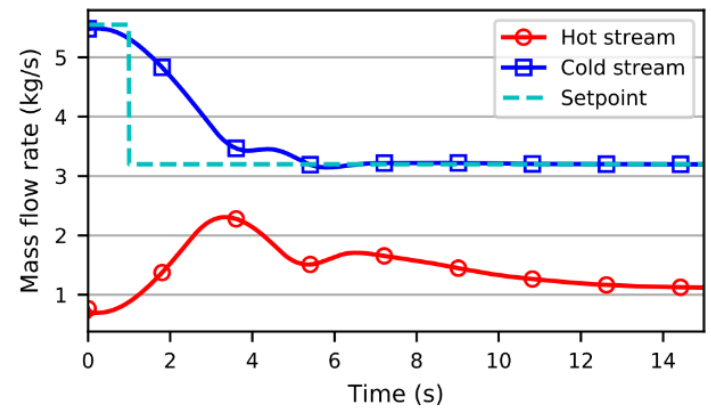

Figure 5 - Closed-loop response of inlet and outlet temperatures and mass flow rates for the offdesign $\mathrm{CO} 2-\mathrm{CO} 2$ case

For the NaK-CO2 case, we simulate the behaviour of the system in response to setpoint changes in cold-stream outlet temperature from $620 \mathrm{~K}$ to $650 \mathrm{~K}$ and cold-stream mass flow rate from $5.2 \mathrm{~kg} / \mathrm{s}$ to $3.8 \mathrm{~kg} / \mathrm{s}$ at $\mathrm{t}=1 \mathrm{sec}$ over $15 \mathrm{sec}$. The temporal profiles of inlet and outlet temperature and mass flow rate for both streams are shown in Fig. 6. (The initial and final temperature distributions are omitted for brevity.)

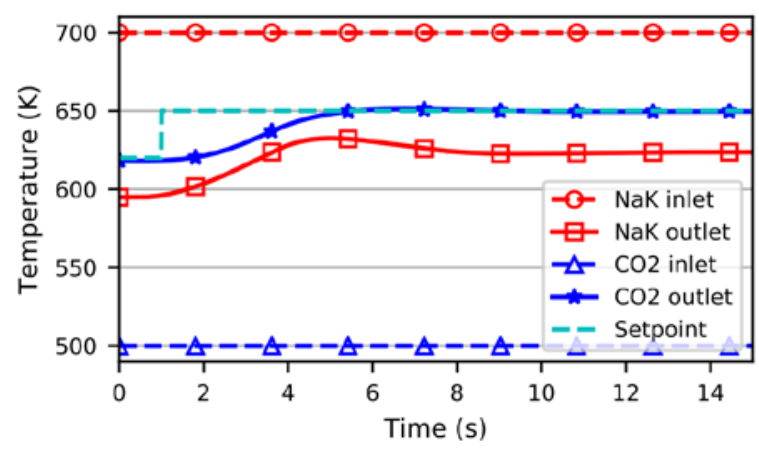

Figure 6 - Closed-loop response of inlet and outlet temperatures and mass flow rates for the NaK-CO2 case

For the design-point case, the controller performs well. The controlled variables are driven to their specified setpoints quickly with no overshoot and no steady-state offset. The rate constraints on the inputs are active through much of the transient, which is a typical characteristic of MPC. For the offdesign case, the controller performs similarly, though slightly overshoots both the mass flow rate and temperature targets. The disturbances are rejected promptly, with almost no impact on the controlled outputs. Again, steady-state offset is negligible $(0.1 \%$ for mass flow rate and $0.06 \%$ for outlet temperature). Similar control characteristics are shown for the NaK-CO2 test case. The excellent closed-loop performance in all test cases suggests that the approach of developing a model predictive controller based on successive online linearisations of the time scale-separated analytical model is valid. The results suggest that MPC may be viable control approach for controlling non-ideal gas heat exchangers in operational processing plants and power cycles, despite the fundamental complexities of these components.

\subsection{Discretisation and model uncertainty study}

MPC is a computationally demanding control methodology. Model complexity is often constrained in 


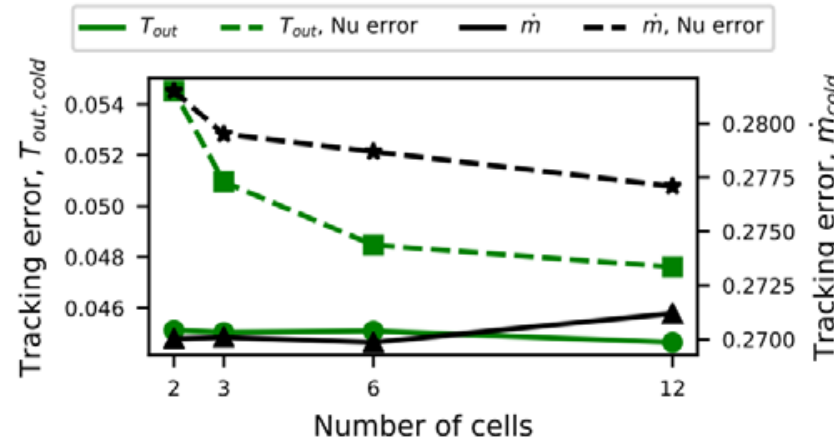

industrial MPC implementations so that real-time operation can be achieved. Here we study the effect of model discretisation on closed-loop performance to gain insight into the computational feasibility of implementing MPC for realworld non-ideal-gas heat exchanger systems.

We repeat the simulations performed for the off-design CO2-CO2 heat exchanger using twelve, six, three, and two cells for both the observer and controller. We analyse controller performance for each discretisation level by computing the normalised squared tracking error for both the temperature and mass flow rate outputs. For the cold-stream outlet temperature, this tracking error is defined as

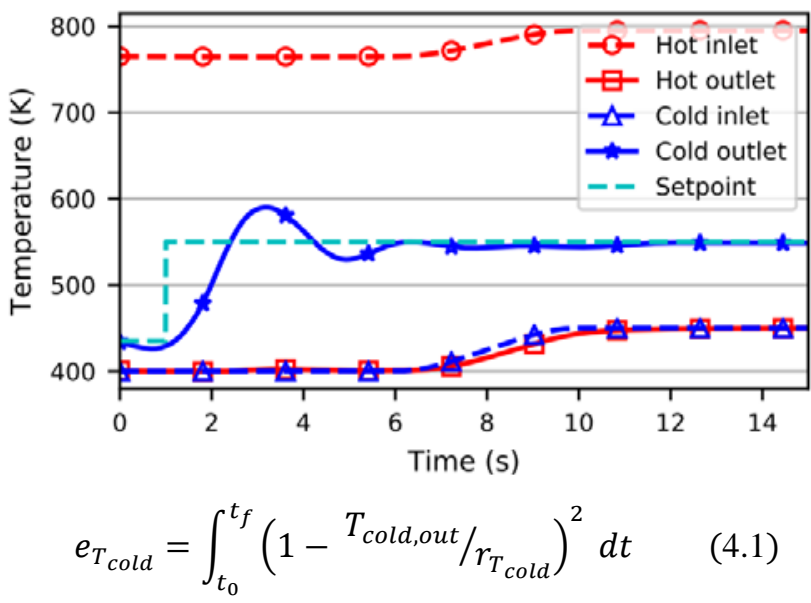

where $r_{T_{c o l d}}$ is the set point value for cold-stream outlet temperature. $e_{m_{\text {cold }}}$, the tracking error for cold-stream mass flow rate is calculated similarly.

Furthermore, for operational non-ideal-gas heat exchangers, exact internal geometry measurements and accurate heat transfer correlations are generally not available (Bone et. al., 2018), leading to errors in heat transfer predictions. Here, we also study the effect of using inaccurate heat transfer correlations in the observer and controller. To study this effect, we scale the Nusselt number in the observer and controller model as

$$
N u_{\text {error }}=N u_{\text {acutal }} \times 0.6,
$$

so that heat transfer is under predicted. We then repeat the off-design $\mathrm{CO} 2-\mathrm{CO} 2$ heat exchanger simulations for all discretisation levels and recompute the tracking errors. The results of these studies are shown in Fig. 7.

The results in Fig. 7 show that good closed-loop performance can be obtained for all discretisation levels when using the true heat transfer model. This result is encouraging for industrial applications, since a comparatively simple and therefore computationally efficient model can be used. However, when using the degraded heat transfer model, controller performance begins to worsen with coarser discretisation.

\section{Figure 7 - Discretisation study using regular (solid line) and degraded (dashed line) models. Closed- loop performance is only sensitive to model discretisation when using a degraded model.}

Fig. 7 suggests that decent controller performance can be obtained in the presence of model uncertainty by using sufficiently-fine discretisation. The temporal profiles of coldstream outlet temperature using a degraded control model for two and twelve cells are shown in Fig 8.

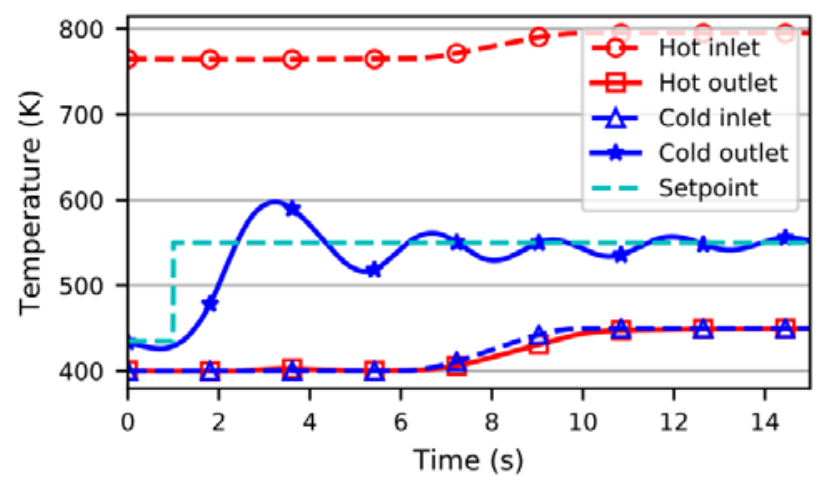

\section{Figure 8 - Inlet and outlet temperature profiles using a degraded control model with 2 cells (top) and 12 cells (bottom)}

The results in Fig. 8 suggest that good performance can be obtained, even in the presence of significant model error, by using sufficiently fine model discretisation. This provides further evidence that MPC is a suitable control methodology for controlling non-ideal-gas heat exchangers.

\section{CONCLUSION}

This paper presented a methodology to implement MPC for heat exchangers with non-ideal-gas working fluids. This methodology involves developing an analytical controloriented heat exchanger model from a high-fidelity simulation model by using timescale separation to capture only the slow dynamics. The MPC is facilitated via successive online linearisations of this control-oriented model, and an observer is used to estimate the internal heat exchanger state from 
inflow and outflow measurements. The controller exhibits good closed-loop performance with quick responses to setpoint changes, prompt disturbance rejection, and negligible steady-state offset. When using an accurate control model, good performance is obtained using a low-dimensional computationally efficient model. When using a control model with significant error, good performance can still be obtained by using sufficient discretisation.

\section{REFERENCES}

Bell, I. H., Wronski, J., Quoilin, S., Lemort, V., 2014. Pure and pseudo-pure fluid thermophysical property evaluation and the open-source thermophysical property library Coolprop. Industrial \& Engineering Chemistry Research 53 (6), 2498-2508.

URL http://pubs.acs.org/doi/abs/10.1021/ie4033999

Bone, V., McNaughton, R., Kearney, M., Jahn, I., 2018. Methodology to develop off-design models of heat exchangers with non-ideal fluids. Applied Thermal Engineering.

Borrelli, F., Bemporad, A. and Morari, M., 2017. Predictive control for linear and hybrid systems. Cambridge University Press.

Carstens, N., 2007. Control strategies for supercritical carbon dioxide power conversion systems. Ph.D. thesis, Massachusetts Institute of Technology.

Courant, R., Friedrichs, K., Lewy, H., 1967. On the partial difference equations of mathematical physics. IBM journal of Research and Development 11 (2), 215-234.

DeCarlo, R. A., 1989. Linear systems: A state variable approach with numerical implementation. Prentice-Hall, Inc.

Dostal, V., Driscoll, M. J., Hejzlar, P., 2004. A supercritical carbon dioxide cycle for next generation nuclear reactors.Ph.D. thesis, Massachusetts Institute of Technology, Department of Nuclear Engineering.

Dubljevic, S., Mhaskar, P., El-Farra, N. H., Christofides, P. D., 2005. Predictive control of transport-reaction processes.Computers \& chemical engineering 29 (11-12), 2335-2345.

Fenghour, A.,Wakeham,W. A., Vesovic, V., 1998. The viscosity of carbon dioxide. Journal of Physical and Chemical Reference Data 27 (1), 31-44.

Franklin, G. F., Powell, J. D., Workman, M. L., 1990. Digital control of dynamic systems. Addison-Wesley Reading, MA, Ch. 2.

Gurobi Optimization, L., 2018. Gurobi optimizer reference manual. URL http://www.gurobi.com

Faghri, A., Zhang, Y. and Howell, J.R., 2010. Advanced heat and mass transfer. Global Digital Press.

Higham, N. J., 2005. The scaling and squaring method for the matrix exponential revisited. SIAM Journal on Matrix

Analysis and Applications 26 (4), 1179-1193.
Issa, R. I., 1986. Solution of the implicitly discretised fluid flow equations by operator-splitting. Journal of computational physics 62 (1), 40-65.

Jacobs, P. A., Gollan, R. J., February 2018. The Eilmer 4.0 flow simulation progrm: Guide to the transient flow solvr, including some examples to get you started. School of Mechanical and Mining Engineering Technical Report 2017/26, The University of Queensland, Brisbane, Australia.

LeVeque, R. J., 2002. Finite volume methods for hyperbolic problems. Vol. 31. Cambridge university press.

Lovera, M., Casella, F., 2015. Model building for control system synthesis. In: Baillieul, J., Samad, T. (Eds.), Encyclopedia of Systems and Control. Springer, pp. 715-722.

Maciejowski, J. M., 2002. Predictive control: with constraints. Pearson education.

Mehos, M., Turchi, C., Jorgenson, J., Denholm, P., Ho, C., Armijo, K., 2016. On the path to sunshot. Advancing concentrating solar power technology, performance, and dispatchability. Tech. rep., National Renewable Energy Laboratory (NREL), Golden, CO (United States).

Moisseytsev, A., Sienicki, J., 2007. Development of a plant dynamics computer code for analysis of a supercritical carbon dioxide brayton cycle energy converter coupled to a natural circulation lead-cooled fast reactor. Tech. rep., Argonne National Laboratory (ANL), Argonne, IL.

Moler, C., Van Loan, C., 2003. Nineteen dubious ways to compute the exponential of a matrix, twenty-five years later. SIAM review 45 (1), 3-49.

Musgrove, G., Sullivan, S., Shiferaw, D., Fourspring, P., Chordia, L., 2017. Heat exchangers. In: Brun, K., Dennis, R. (Eds.), Fundamentals and Applications of Supercritical Carbon Dioxide (sCO2) Based Power Cycles. Woodhead Publishing in energy. Elsevier Science \& Technology, Ch. 8, pp. 217-244.

Musgrove, G., Wright, S., 2017. Introduction and background. In: Brun, K., Dennis, R. (Eds.), Fundamentals and Applications of Supercritical Carbon Dioxide (sCO2) Based Power Cycles. Woodhead Publishing in energy. Elsevier Science \& Technology, Ch. 1, pp. 1-21.

Ngo, T.L., Kato, Y., Nikitin, K. and Ishizuka, T., 2007. Heat transfer and pressure drop correlations of microchannel heat exchangers with S-shaped and zigzag fins for carbon dioxide cycles. Experimental Thermal and Fluid Science, 32(2), pp.560-570.

Poinsot, T. J., Lelef, S., 1992. Boundary conditions for direct simulations of compressible viscous flows. Journal of computational physics 101 (1), 104-129.

Roe, P. L., 1986. Characteristic-based schemes for the euler equations. Annual review of fluid mechanics 18 (1), 337-365.

Scalabrin, G., Marchi, P., Finezzo, F., Span, R., 2006. A reference multiparameter thermal conductivity equation for 
carbon dioxide with an optimized functional form. Journal of physical and chemical reference data 35 (4), 1549-1575.

Span, R., Wagner, W., 1996. A new equation of state for carbon dioxide covering the fluid region from the triplepoint temperature to $1100 \mathrm{k}$ at pressures up to $800 \mathrm{mpa}$. Journal of physical and chemical reference data 25 (6), 1509-1596.

Turchi, C. S., Ma, Z., Neises, T. W., Wagner, M. J., 2013. Thermodynamic study of advanced supercritical carbon dioxide power cycles for concentrating solar power systems. Journal of Solar Energy Engineering 135 (4), 041007.

Versteeg, H., Malalasekera, W., 2007a. An introduction to computational fluid dynamics: the finite volume method. Prentice Hall, Ch. Solution algorithms for pressure-velocity coupling in steady flows.

Versteeg, H., Malalasekera, W., 2007b. An introduction to computational fluid dynamics: the finite volume method. Prentice Hall, Ch. The finite volume method for convectiondiffusion problems.

Wada, Y., Liou, M.-S., 1997. An accurate and robust flux splitting scheme for shock and contact discontinuities. SIAM Journal on Scientific Computing 18 (3), 633-657.

Wang, Y., Boyd, S., 2010. Fast model predictive control using online optimization. IEEE Transactions on control systems technology 18 (2), 267-278.

White, F., 2011. Fluid Mechanics. McGraw-Hill series in mechanical engineering. McGraw Hill. URL https://books.google.com.au/books?id = egk8SQAACAAJ

Zavoico, A. B., 2001. Solar power tower design basis document. Tech. rep., Sandia National Laboratories.

\section{APPENDIX A - COPYRIGHT/OPEN ACCESS}

The GPPS policy is that all articles will be Open Source accessible. This article will be published using the Creative Commons Attribution CC-BY 4.0, thus allowing the author(s) to retain their copyright.

For answers to frequently asked questions about Creative

Commons Licences, please see

https://creativecommons.org/faq/.

\section{APPENDIX B - GPPS Presenter Policy and Paper Acceptance}

According to GPPS's presenter attendance policy, a paper cannot be published or be indexed and may not be cited as a published paper until at least one author pays the registration fee and attends the conference. The GPPS reserves the right to withdraw from its publications any paper that is not presented by an author of the paper at the appropriate conference. Any paper that is withdrawn may not be cited as a published paper. 